\title{
ON RELATIONS BETWEEN ENTROPY AND HAUSDORFF DIMENSION OF MEASURES *
}

\author{
ATHANASIOS BATAKIS ${ }^{\dagger}$ AND YANICK HEURTEAUX ${ }^{\ddagger}$
}

\begin{abstract}
We characterize probability measures whose Hausdorff dimension or packing dimension can be calculated by an entropy formula. In particular, we prove that such measures are unidimensional. We also construct examples of unidimensional measures for which entropy does not calculate the dimension.
\end{abstract}

Let $D$ be an integer greater than 1 and $m$ be a probability measure in $[0,1)^{D}$. Fix $\ell \geq 1$ and denote by $\mathcal{F}_{n}$ the family of $\ell$-adic cubes of the $n^{\text {th }}$ generation, that is

$$
\mathcal{F}_{n}=\left\{I=\prod_{i=1}^{D}\left[k_{i} / \ell^{n},\left(k_{i}+1\right) / \ell^{n}\right) ; 0 \leq k_{i}<\ell^{n}\right\} .
$$

For an arbitrary point $x$ in $[0,1)^{D}$, let $I_{n}(x)$ be the unique cube $I \in \mathcal{F}_{n}$ such that $x \in I$.

We want to give estimates for the lower and the upper dimension of the measure $m$. These are respectively defined by

$$
\left\{\begin{array}{l}
\operatorname{dim}_{*}(m)=\inf (\operatorname{dim}(E) ; m(E)>0) \\
\operatorname{dim}^{*}(m)=\inf (\operatorname{dim}(E) ; m(E)=1)
\end{array} .\right.
$$

It is well known that there exist some relations between these quantities and the function $\tau$ which appears in the multifractal formalism. More precisely, if we let :

$$
\tau_{n}(t)=\frac{1}{n \log \ell} \log \left(\sum_{I \in \mathcal{F}_{n}} m(I)^{t}\right) \quad \text { and } \quad \tau(t)=\limsup _{n \rightarrow+\infty} \tau_{n}(t)
$$

it is proved in [11] that

$$
-\tau_{+}^{\prime}(1) \leq \operatorname{dim}_{*}(m) \leq h_{*}(m)
$$

where $h_{*}(m)$ is the lower entropy (also called lower Rényi dimension) of the measure $m$, defined as

$$
h_{*}(m)=\liminf _{n \rightarrow+\infty} h_{n}(m) \quad \text { where } \quad h_{n}(m)=-\tau_{n}^{\prime}(1)=\frac{-1}{n \log \ell} \sum_{I \in \mathcal{F}_{n}} m(I) \log (m(I)) .
$$

In [11], we also give sufficient conditions for the equality $-\tau_{+}^{\prime}(1)=\operatorname{dim}_{*}(m)$ to hold. In this paper we are interested in describing the measures $m$ satisfying $\operatorname{dim}_{*}(m)=$ $h_{*}(m)$. Theorem 1.1 states that $\operatorname{dim}_{*}(m)=h_{*}(m)$ holds if and only if there exists a subsequence $n_{k}$ such that

$$
\lim _{k \rightarrow+\infty} \frac{\log m\left(I_{n_{k}}(x)\right)}{-n_{k} \log \ell}=\operatorname{dim}_{*}(m) \quad d m \text {-almost surely } .
$$

\footnotetext{
*Received August 19, 2001; accepted for publication May 7, 2002.

†Département de Mathématiques, Université d'Orléans, B.P. 6759, F-45067 Orléans cedex 2, France (Athanasios.Batakis@labomath.univ-orleans.fr).

${ }^{\ddagger}$ Laboratoire de Mathématiques pures, Université Blaise Pascal, F-63177 Aubière cedex, France (Yanick.Heurteaux@math.univ-bpclermont.fr).
} 
In particular, such measures are unidimensional (i.e. satisfy $\operatorname{dim}_{*}(m)=\operatorname{dim}^{*}(m)$ ). If we denote by $\operatorname{dim}(m)$ this common value, the measure $m$ is supported by a set of dimension $\operatorname{dim}(m)$ but every set of dimension strictly less than $\operatorname{dim}(m)$ is negligible. Nevertheless, unidimensionality is not a sufficient condition to have $\operatorname{dim}_{*}(m)=h_{*}(m)$. An example of a measure of exact dimension (i.e. unidimensional) for which $\operatorname{dim}_{*}(m)<h_{*}(m)$ is proposed in Proposition 3.1.

The interest of such a result is that it is not written in a dynamical context. Nevertheless, the conclusion (2) can be interpreted as a weak form of a ShannonMcMillan type result.

Similar results can be established, comparing the upper entropy

$$
h^{*}(m)=\limsup _{n \rightarrow+\infty} h_{n}(m)
$$

and the packing dimension of the measure $m$. Following [13], [11] or [8], we can introduce

$$
\left\{\begin{array}{l}
\operatorname{Dim}_{*}(m)=\inf (\operatorname{Dim}(E) ; m(E)>0) \\
\operatorname{Dim}^{*}(m)=\inf (\operatorname{Dim}(E) ; m(E)=1)
\end{array},\right.
$$

where $\operatorname{Dim}(E)$ is the packing dimension of the set $E$ (for more details on packing dimension, see [7] or the original paper of Tricot [14]). As proved in [11],

$$
h^{*}(m) \leq \operatorname{Dim}^{*}(m) \leq-\tau_{-}^{\prime}(1)
$$

and we characterize in Theorem 1.2 of the present paper the measures for which the equality $h^{*}(m)=\operatorname{Dim}^{*}(m)$ holds.

Several examples of measures satisfying $\operatorname{dim}_{*}(m)=h_{*}(m)$ and $\operatorname{Dim}^{*}(m)=h^{*}(m)$ are also proposed. In particular, this is the case in an ergodic situation (Example 1.3), for quasi-Bernoulli measures (Example 1.5) and in a context where the strong law of large numbers can be applied (Example 1.6).

In the last section, we observe that the behaviour related to the Hausdorff dimension and the packing dimension can be different. We construct a measure $m$ of exact dimension for which

$$
\operatorname{dim}(m)=h_{*}(m) \quad \text { but } \quad \operatorname{Dim}(m)>h^{*}(m) .
$$

1. Main results and examples. The main result of this paper is the following.

THEOREM 1.1. Let $m$ be a probability measure in $[0,1)^{D}$. Then

$$
\operatorname{dim}_{*}(m) \leq h_{*}(m) .
$$

Moreover, the following properties are equivalent :

(i) $\operatorname{dim}_{*}(m)=h_{*}(m)$

(ii) $\operatorname{dim}_{*}(m)=\operatorname{dim}^{*}(m)=h_{*}(m)$

(iii) There exists a subsequence $\left(n_{k}\right)_{k \geq 1}$ such that for dm-almost every $x \in[0,1)^{D}$,

$$
\lim _{k \rightarrow+\infty} \frac{\log m\left(I_{n_{k}}(x)\right)}{-n_{k} \log \ell}=\operatorname{dim}_{*}(m) .
$$

A similar result can also be established, comparing the upper entropy $h^{*}(m)$ of a measure $m$ with its packing dimension $\operatorname{Dim}^{*}(m)$. 
THEOREM 1.2. We also have

$$
h^{*}(m) \leq \operatorname{Dim}^{*}(m)
$$

and the following properties are equivalent:

(i) $\operatorname{Dim}^{*}(m)=h^{*}(m)$

(ii) $\operatorname{Dim}_{*}(m)=\operatorname{Dim}^{*}(m)=h^{*}(m)$

(iii) There exists a subsequence $\left(n_{k}\right)_{k \geq 1}$ such that for dm-almost every $x \in[0,1)^{D}$,

$$
\lim _{k \rightarrow+\infty} \frac{\log m\left(I_{n_{k}}(x)\right)}{-n_{k} \log \ell}=\operatorname{Dim}^{*}(m)
$$

REMARK. As was pointed out in the introduction, unidimensionality is not sufficient to establish the equalities $\operatorname{dim}_{*}(m)=h_{*}(m)$ and $\operatorname{Dim}^{*}(m)=h^{*}(m)$ (see Propositions 3.1 and 4.1). In fact, the statements of Theorems 1.1 and 1.2 stem from a deep homogeneity property.

Let us now give some useful examples of measures $m$ for which the equalities $\operatorname{dim}_{*}(m)=h_{*}(m)$ and $\operatorname{Dim}^{*}(m)=h^{*}(m)$ hold.

EXAMPLE 1.3. Suppose that the sequence

$$
\frac{\log m\left(I_{n}(x)\right)}{-n \log \ell}
$$

converges almost surely to a constant $d$. Then, the equivalent properties of Theorems 1.1 and 1.2 are satisfied and we have

$$
d=\operatorname{dim}(m)=\operatorname{Dim}(m)
$$

In particular, this is the case in an ergodic context. Let us denote the elements of $\mathcal{F}_{n}$ by $I_{\varepsilon_{1}, \ldots, \varepsilon_{n}}$ with $\varepsilon_{i} \in\left\{0, \ldots, \ell^{D}-1\right\}$ in such a way that $I_{\varepsilon_{1}, \ldots, \varepsilon_{n+1}} \subset I_{\varepsilon_{1}, \ldots, \varepsilon_{n}}$. Define $J(x)$ to be the unique element $\left(\varepsilon_{i}\right)_{i>1}$ of the Cantor set $\left\{0, \ldots, \ell^{D}-1\right\}^{\mathbb{N}^{*}}$ such that $\{x\}=\bigcap_{n} I_{\varepsilon_{1}, \ldots, \varepsilon_{n}}$, and consider the image $\tilde{m}$ of $m$ with respect to the application $J$. If we suppose that the mesure $\tilde{m}$ is invariant and ergodic with respect to the shift operator, Shannon-McMillan's theorem ensures that (4) admits an almost sure constant limit $d$ and that (5) is satisfied (see [11] for more details and [16] for basic facts on ergodic theory).

Example 1.4. The following situation was described by S.M. Ngai in [12]. Suppose that the function $\tau$ of the multifractal formalism admits a derivative $\tau^{\prime}(1)$. Then, using inequalities (1) and (3), we conclude that the measure $m$ is unidimensional and satisfies

$$
\operatorname{dim}(m)=\operatorname{Dim}(m)=h(m)=-\tau^{\prime}(1)
$$

where $h(m)=h_{*}(m)=h^{*}(m)$ is the genuine limit of the sequence $h_{n}(m)$.

ExAmple 1.5. The case of quasi-Bernoulli measures is related to examples 1.3 and 1.4. Suppose that there exists a constant $C>0$ such that for every $\varepsilon_{1}, \ldots, \varepsilon_{n+p}$ we have :

$\frac{1}{C} m\left(I_{\varepsilon_{1}, \ldots, \varepsilon_{n}}\right) m\left(I_{\varepsilon_{n+1}, \ldots, \varepsilon_{n+p}}\right) \leq m\left(I_{\varepsilon_{1}, \ldots, \varepsilon_{n+p}}\right) \leq C m\left(I_{\varepsilon_{1}, \ldots, \varepsilon_{n}}\right) m\left(I_{\varepsilon_{n+1}, \ldots, \varepsilon_{n+p}}\right)$. 
It is well known (see [6]) that such a measure is equivalent to an invariant and ergodic measure. Moreover, it is proved in [11] that $\tau^{\prime}(1)$ exists in this case. Let us also remember that the multifractal formalism is available for such measures (see [5] and [11]).

EXAMPLE 1.6. Let us write

$$
X_{n}(x)=\log \left(\frac{m\left(I_{n}(x)\right)}{m\left(I_{n-1}(x)\right)}\right)
$$

and suppose that

$$
\lim _{n \rightarrow+\infty} \frac{1}{n} \sum_{i=1}^{n}\left(X_{i}-\mathbb{E}\left(X_{i}\right)\right)=0 \text { dm a.s. }
$$

where the expectation is related to the probability $m$. If we remark that

$$
h_{n}(m)=\frac{-1}{n \log \ell} \sum_{i=1}^{n} \mathbb{E}\left(X_{i}\right)
$$

we can easily conclude that the equalities $\operatorname{dim}_{*}(m)=h_{*}(m)$ and $\operatorname{Dim}^{*}(m)=h^{*}(m)$ are satisfied in this situation. In particular, this is the case when the random variables $X_{i}$ are bounded in $L^{2}$ and uncorrelated.

A classical case (called Bernoulli product) of such measures is often described in the literature (see for example [4], [3] or [11]). Fix a sequence $\left(p_{i}\right)_{i \geq 1}$ of real numbers with $0<p_{i}<1$ and consider a sequence $\left(Y_{i}\right)_{i \geq 1}$ of independant 0,1 -valued random variables such that

$$
\mathbb{P}\left(Y_{i}=0\right)=p_{i} \quad \text { and } \quad \mathbb{P}\left(Y_{i}=1\right)=1-p_{i} .
$$

Then, the law $m$ of the random variable $\sum_{i=1}^{+\infty} 2^{-i} Y_{i}$ satisfies

$$
\left\{\begin{array}{l}
\operatorname{dim}(m)=h_{*}(m)=\liminf _{n \rightarrow+\infty} \frac{1}{n} \sum_{i=1}^{n} s\left(p_{i}\right) \\
\operatorname{Dim}(m)=h^{*}(m)=\limsup _{n \rightarrow+\infty} \frac{1}{n} \sum_{i=1}^{n} s\left(p_{i}\right)
\end{array}\right.
$$

where the function $s(t)$ is defined by

$$
s(t)=-\left[t \log _{2} t+(1-t) \log _{2}(1-t)\right] \text { for } t \in[0,1]
$$

and $\log _{2}$ is the logarithm in base 2 .

2. Proof of Theorems 1.1 and 1.2. We prove Theorem 1.1. Since the proof of Theorem 1.2 is similar, we will only sketch it at the end of the section.

The inequality $\operatorname{dim}_{*}(m) \leq h_{*}(m)$ is well known (see for example [11] or [15]). Nevertheless, let us give an elementary proof in order to make easier the study of the equality case. If $x \in[0,1)^{D}$, put :

$$
\alpha_{n}(x)=\frac{\log m\left(I_{n}(x)\right)}{-n \log \ell} \quad \text { and } \quad \underline{\alpha}(x)=\liminf _{n \rightarrow+\infty} \alpha_{n}(x) .
$$


It is well known ([9], [8] or [11]) that :

$$
\operatorname{dim}_{*}(m)=\sup (\{\alpha \geq 0 ; \underline{\alpha} \geq \alpha \text { dm a.s. }\})=\inf \operatorname{ess}(\underline{\alpha}) .
$$

We also observe that $h_{n}(m)=\int \alpha_{n}(x) d m(x)$. Using Fatou's lemma and the fact that $m$ is a probability measure, we get

$$
\operatorname{dim}_{*}(m) \leq \int \underline{\alpha}(x) d m(x) \leq h_{*}(m) .
$$

Proof of (iii) $\Rightarrow$ (i). Suppose that there exists a subsequence $\left(n_{k}\right)_{k \geq 1}$ such that for $d m$-almost every $x \in[0,1)^{D}$,

$$
\lim _{k \rightarrow+\infty} \alpha_{n_{k}}(x)=\operatorname{dim}_{*}(m) .
$$

Using the dominated convergence theorem (see the domination $\phi$ below), we obtain

$$
\operatorname{dim}_{*}(m)=\int \lim _{k \rightarrow+\infty} \alpha_{n_{k}}(x) d m(x)=\lim _{k \rightarrow+\infty} h_{n_{k}}(m) \geq h_{*}(m)
$$

and we are done.

Lemma 2.1. Let $\phi=\sup _{n \geq 1} \alpha_{n}$. Then $\phi \in L^{1}(m)$.

Proof. It is sufficient to prove that the real variable function

$$
t \longmapsto m(\{x ; \phi(x)>t\})
$$

is integrable in a neighbourhood of $+\infty$ with respect to the Lebesgue's measure. But we know that $\alpha_{n}(x)>t$ if and only if $m\left(I_{n}(x)\right)<\ell^{-n t}$. According to the fact that the partition $\mathcal{F}_{n}$ contains $\ell^{n D}$ elements,

$$
m\left(\left\{x ; \alpha_{n}(x)>t\right\}\right) \leq \ell^{n D} \ell^{-n t} .
$$

If $t>D$, we obtain

$$
m(\{x ; \phi(x)>t\}) \leq \sum_{n=1}^{+\infty} m\left(\left\{x ; \alpha_{n}(x)>t\right\}\right) \leq \frac{\ell^{D-t}}{1-\ell^{D-t}}
$$

which proves the integrability of $\phi$.

Proof of (i) $\Rightarrow$ (ii). Suppose that $\operatorname{dim}_{*}(m)=h_{*}(m)$. Using (7) and (8), we remark that for $d m$-almost every point $x, \underline{\alpha}(x)=\operatorname{dim}_{*}(m)$. Then, we also have

$$
\operatorname{dim}_{*}(m)=\inf (\{\alpha \geq 0 ; \underline{\alpha} \leq \alpha \text { dm a.s. }\})=\sup \operatorname{ess}(\underline{\alpha})
$$

and hence $\operatorname{dim}_{*}(m)=\operatorname{dim}^{*}(m)$ (see [11] or [8]). The measure $m$ is thus unidimensional.

Proof of (ii) $\Rightarrow$ (iii). Let $d=\operatorname{dim}_{*}(m)=h_{*}(m)$. We will make use of the following lemma.

LEMMA 2.2. Let $\eta \in(0,1)$ and $n_{0} \geq 1$. We can choose an integer $n_{1} \geq n_{0}$ such that :

$$
m\left(\left\{x ; \alpha_{n_{1}}>d+\eta\right\}\right) \leq(2+d) \eta
$$


Proof. As was remarked before, the equality $\underline{\alpha}=d$ holds $d m$-almost surely. We can then find $n_{0}^{\prime} \geq n_{0}$ such that

$$
m\left(\bigcap_{n \geq n_{0}^{\prime}}\left\{x ; \alpha_{n}(x)>d-\eta^{2}\right\}\right)>1-\eta^{2} .
$$

On the other hand, by hypothesis (ii), we can choose an integer $n_{1} \geq n_{0}^{\prime}$ such that

$$
h_{n_{1}}(m)=\int \alpha_{n_{1}}(x) \cdot d m(x)<d+\eta^{2} .
$$

This integer $n_{1}$ will be the right one. Let us denote

$$
A=\left\{x ; \alpha_{n_{1}}(x)>d-\eta^{2}\right\} \quad \text { and } \quad B=\left\{x ; \alpha_{n_{1}}(x)>d+\eta\right\} .
$$

We have

$$
\begin{aligned}
d+\eta^{2} & \geq \int \alpha_{n_{1}}(x) d m(x) \\
& \geq \int_{A \backslash B} \alpha_{n_{1}}(x) d m(x)+\int_{B} \alpha_{n_{1}}(x) d m(x) \\
& \geq\left(d-\eta^{2}\right)(m(A)-m(B))+(d+\eta) m(B) .
\end{aligned}
$$

If we remember that $m(A) \geq 1-\eta^{2}$, we get

$$
m(B) \leq \frac{2 \eta^{2}+d \eta^{2}}{\eta+\eta^{2}} \leq(2+d) \eta
$$

which is the conclusion of the lemma.

By using Lemma 2.2 with $\eta=2^{-k}$, we can construct a subsequence $\left(n_{k}\right)_{k \geq 1}$ such that for all $k \geq 1$,

$$
m\left(\left\{x ; \alpha_{n_{k}}(x)>d+2^{-k}\right\}\right) \leq(2+d) 2^{-k} .
$$

By applying Borel Cantelli's lemma, we then obtain

$$
\limsup _{k \rightarrow+\infty} \alpha_{n_{k}}(x) \leq d \quad d m \text { a.s. . }
$$

On the other hand,

$$
d=\underline{\alpha}(x) \leq \liminf _{k \rightarrow+\infty} \alpha_{n_{k}}(x) \text { dm a.s. . }
$$

Thus, we have proved that the subsequence $\left(\alpha_{n_{k}}\right)_{k \geq 1}$ converges almost surely to $d$.

To prove Theorem 1.2, we introduce the function

$$
\bar{\alpha}(x)=\limsup _{n \rightarrow+\infty} \alpha_{n}(x) .
$$

As proved in [8], [11] and [13], we have :

$$
\operatorname{Dim}^{*}(m)=\inf (\{\alpha \geq 0 ; \bar{\alpha} \leq \alpha d m \text { a.s. }\})=\sup \operatorname{ess}(\bar{\alpha}) \text {. }
$$

The inequality $h^{*}(m) \leq \operatorname{Dim}^{*}(m)$ is a consequence of Fatou's lemma applied to the sequence $\phi-\alpha_{n}$. The study of the equality case uses the same ideas as before. 
3. An example where $\operatorname{dim}(m)<h_{*}(m)$ and $\operatorname{Dim}(m)>h^{*}(m)$. In this section, we take $D=1$ and $\ell=2$. We begin with the construction of a family of auxiliary measures which are of the type described in Example 1.6. Let $a$ and $b$ be two real numbers with $0<a, b<1$ and fix a sequence of integers $\left(T_{k}\right)_{k \geq 1}$ such that

$$
T_{1}=1, \quad T_{k}<T_{k+1} \text { and } \lim _{k \rightarrow+\infty} \frac{T_{k+1}}{T_{k}}=+\infty .
$$

Then, we define the family of parameters $p_{a b}(i)$ :

$$
p_{a b}(i)=a \text { if } T_{2 n-1} \leq i<T_{2 n} \quad \text { and } \quad p_{a b}(i)=b \text { if } T_{2 n} \leq i<T_{2 n+1} .
$$

If $\left(Y_{i}\right)_{i \geq 1}$ is a sequence of independant random variables such that

$$
\mathbb{P}\left(Y_{i}=0\right)=p_{a b}(i) \quad \text { and } \quad \mathbb{P}\left(Y_{i}=1\right)=1-p_{a b}(i)
$$

we denote by $m_{a b}$ the law of the random variable $\sum_{i=1}^{+\infty} 2^{-i} Y_{i}$.

The choice of the integers $T_{k}$ and the identities (6) ensure that for $d m_{a b}$-almost every $x \in[0,1)$, we have :

$$
\left\{\begin{array}{l}
\liminf _{n \rightarrow+\infty} \frac{\log m_{a b}\left(I_{n}(x)\right)}{-n \log 2}=\inf (s(a), s(b)) \\
\limsup _{n \rightarrow+\infty} \frac{\log m_{a b}\left(I_{n}(x)\right)}{-n \log 2}=\sup (s(a), s(b))
\end{array} .\right.
$$

We can now construct our counterexamples. Let us fix a parameter $\beta \in(0,1 / 2]$ and two real numbers $p$ and $\tilde{p}$ such that $0<p<\tilde{p} \leq 1 / 2$. If $F(t)$ is defined by

$$
F(t)=2 t \text { when } t \in[0,1 / 2) \text { and } F(t)=2 t-1 \text { when } t \in[1 / 2,1),
$$

we are interested in the measure $m_{\beta}$ defined by

$$
m_{\beta}(A)=\beta m_{p \tilde{p}}(F([0,1 / 2) \cap A))+(1-\beta) m_{\tilde{p} p}(F([1 / 2,1) \cap A)) .
$$

In other words, the measure $m_{\beta}$ assigns the mass $\beta$ (resp. $1-\beta$ ) to the interval $[0,1 / 2)$ (resp. $[1 / 2,1))$ and is a copy, in this set, of the measure $m_{p \tilde{p}}$ (resp. $m_{\tilde{p} p}$ )

Measures $m_{\beta}$ are examples of unidimensional measures whose dimension can not be calculated with an entropy formula. More precisely, we have the following.

Proposition 3.1. The measure $m_{\beta}$ satisfies the following properties :

(i) $\operatorname{dim}_{*}\left(m_{\beta}\right)=\operatorname{dim}^{*}\left(m_{\beta}\right)=s(p)$

(ii) $\operatorname{Dim}_{*}\left(m_{\beta}\right)=\operatorname{Dim}^{*}\left(m_{\beta}\right)=s(\tilde{p})$

(iii) $h_{*}\left(m_{\beta}\right)=\beta s(\tilde{p})+(1-\beta) s(p) \quad$ and $h^{*}\left(m_{\beta}\right)=\beta s(p)+(1-\beta) s(\tilde{p})$. In particular, $\operatorname{dim}\left(m_{\beta}\right)<h_{*}\left(m_{\beta}\right)$ and $h^{*}\left(m_{\beta}\right)<\operatorname{Dim}\left(m_{\beta}\right)$.

REMARKS. 1. For such a measure $m_{\beta}$, unidimensionality ensures that for almost every point $x$, there exists a subsequence $n_{k}$ such that $\alpha_{n_{k}}(x)$ converges to $\operatorname{dim}_{*}\left(m_{\beta}\right)$. Nevertheless, according to Theorem 1.1, we can not find a subsequence $n_{k}$ such that $\alpha_{n_{k}}$ converges almost surely to $\operatorname{dim}_{*}\left(m_{\beta}\right)$. A similar remark can be made concerning $\operatorname{Dim}^{*}\left(m_{\beta}\right)$.

2. In the case where $\beta=1 / 2$, we obtain a unidimensional measure $m$ (in the Hausdorff and in the packing sense), which posseses an entropy $h(m)$ (i.e. $h_{*}(m)=$ $\left.h^{*}(m)=h(m)\right)$, but verifies $\operatorname{dim}(m)<h(m)<\operatorname{Dim}(m)$. 
3. If $\beta_{1} \neq \beta_{2}$, we obtain two measures $\mu=m_{\beta_{1}}$ and $\nu=m_{\beta_{2}}$ which are unidimensional, strongly equivalent (i.e. $\frac{1}{c} \nu \leq \mu \leq c \nu$ ), but satisfy $h_{*}(\mu) \neq h_{*}(\nu)$ and $h^{*}(\mu) \neq h^{*}(\nu)$. This result indicates that entropy is a bad concept of dimension (even for unidimensional measures) ; for a good concept of dimension, it is indeed reasonable to demand that two equivalent measures should have the same dimension.

Proof of Proposition 3.1. Properties 1 and 2 are immediate consequences of (9). We only have to prove property 3 . Let us put $p_{i}=p_{p \tilde{p}}(i)$ and $\tilde{p}_{i}=p_{\tilde{p} p}(i)$. We have

$$
\tau_{n}(t)=\frac{1}{n} \log _{2}\left(\beta^{t} \prod_{i=1}^{n-1}\left(p_{i}^{t}+\left(1-p_{i}\right)^{t}\right)+(1-\beta)^{t} \prod_{i=1}^{n-1}\left(\tilde{p}_{i}^{t}+\left(1-\tilde{p}_{i}\right)^{t}\right)\right) .
$$

If we remember that $h_{n}\left(m_{\beta}\right)=-\tau_{n}^{\prime}(1)$, we obtain

$$
h_{n}\left(m_{\beta}\right)=\frac{1}{n}\left(\beta\left(-\log _{2} \beta+\sum_{i=1}^{n-1} s\left(p_{i}\right)\right)+(1-\beta)\left(-\log _{2}(1-\beta)+\sum_{i=1}^{n-1} s\left(\tilde{p}_{i}\right)\right)\right) .
$$

If $n_{0}(n)$ is the number of integers $i \leq n-1$ such that $p_{i}=p$ and if $n_{1}(n)=n-1-n_{0}(n)$, we can then write

$$
h_{n}\left(m_{\beta}\right)=\frac{n_{0}(n)}{n}(\beta s(p)+(1-\beta) s(\tilde{p}))+\frac{n_{1}(n)}{n}(\beta s(\tilde{p})+(1-\beta) s(p))+O\left(\frac{1}{n}\right) .
$$

In particular, we have

$$
\beta s(\tilde{p})+(1-\beta) s(p)+O\left(\frac{1}{n}\right) \leq h_{n}\left(m_{\beta}\right) \leq \beta s(p)+(1-\beta) s(\tilde{p})+O\left(\frac{1}{n}\right) .
$$

To conclude, it suffices to remark that

$$
\limsup _{n \rightarrow+\infty} \frac{n_{0}(n)}{n}=1 \text { and } \limsup _{n \rightarrow+\infty} \frac{n_{1}(n)}{n}=1
$$

4. An example where $\operatorname{dim}(m)=h_{*}(m)$ but $\operatorname{Dim}(m)>h^{*}(m)$. As observed in Example 1.6, the equalities $\operatorname{dim}_{*}(m)=h_{*}(m)$ and $\operatorname{Dim}^{*}(m)=h^{*}(m)$ hold as soon as the sequence of random variables

$$
X_{n}(x)=\log \left(\frac{m\left(I_{n}(x)\right)}{m\left(I_{n-1}(x)\right)}\right)
$$

satisfies the strong law of large numbers

$$
\lim _{n \rightarrow+\infty} \frac{1}{n} \sum_{i=1}^{n}\left(X_{i}-\mathbb{E}\left(X_{i}\right)\right)=0 \text { dm a.s. }
$$

We are going to construct a unidimensional measure $m$ for which $\operatorname{dim}_{*}(m)=h_{*}(m)$ but $\operatorname{Dim}^{*}(m)>h^{*}(m)$. Of course, such a measure does not satisfy (11).

Proposition 4.1. There exists a unidimensional probability measure $m$ such that

$$
\operatorname{dim}_{*}(m)=\operatorname{dim}^{*}(m)=h_{*}(m) \quad \text { but } \quad h^{*}(m)<\operatorname{Dim}^{*}(m)=\operatorname{Dim}_{*}(m) .
$$


The notations are the same as in the previous section. Let $\beta=1 / 2$. The measure $\mu_{1}=m_{p \tilde{p}}$ is the same as before but we modify slightly the measure $m_{\tilde{p} p}$. In section 3 , the measure $m_{\tilde{p} p}$ was constructed in such a way that

$$
\forall n \geq 1, \forall i \in\left\{T_{2 n-1}, \ldots, T_{2 n}-1\right\}, \quad \tilde{p}_{i}=p_{\tilde{p} p}(i)=\tilde{p}
$$

We modify the value of $\tilde{p}_{i}$ according to the parity of $n$ and define

$$
\left\{\begin{array}{l}
\tilde{p}_{i}=\tilde{p} \text { if } i \in\left\{T_{2 n-1}, \ldots, T_{2 n}-1\right\} \quad \text { and } n=2 k+1 \\
\tilde{p}_{i}=p \text { if } i \in\left\{T_{2 n-1}, \ldots, T_{2 n}-1\right\} \quad \text { and } n=2 k
\end{array} .\right.
$$

We do not modify the value of $\tilde{p}_{i}$ when $i \in\left\{T_{2 n}, \ldots, T_{2 n+1}-1\right\}$. Let $\mu_{2}$ be the so constructed measure and put :

$$
m(A)=\frac{1}{2} \mu_{1}(F([0,1 / 2) \cap A))+\frac{1}{2} \mu_{2}(F([1 / 2,1) \cap A)) .
$$

Similar computations as those made before ensure that :

$$
\operatorname{dim}_{*}(m)=\operatorname{dim}^{*}(m)=s(p) \text { and } \operatorname{Dim}_{*}(m)=\operatorname{Dim}^{*}(m)=s(\tilde{p}) .
$$

Let us see the evolutions of entropy. Since $p<\tilde{p}$, the new measure has smaller entropy than the measure $m_{1 / 2}$ (corresponding to the parameter $\beta=1 / 2$ ) of Proposition 3.1. Hence,

$$
h^{*}(m) \leq \frac{1}{2}(s(p)+s(\tilde{p}))<\operatorname{Dim}^{*}(m)
$$

In fact, it is easy to prove that $h^{*}(m)=(s(p)+s(\tilde{p})) / 2$.

Moreover, using formula (10) for the new measure $m$, we have :

$$
\begin{aligned}
h_{T_{4 k}}(m) & =\frac{1}{2 T_{4 k}} \sum_{i=1}^{T_{4 k}-1}\left(s\left(p_{i}\right)+s\left(\tilde{p}_{i}\right)\right)+O\left(\frac{1}{T_{4 k}}\right) \\
& =\frac{T_{4 k}-T_{4 k-1}}{T_{4 k}} \cdot s(p)+O\left(\frac{T_{4 k-1}}{T_{4 k}}\right)+O\left(\frac{1}{T_{4 k}}\right) .
\end{aligned}
$$

It follows that

$$
h_{*}(m) \leq \lim _{k \rightarrow+\infty} h_{T_{4 k}}(m)=s(p)=\operatorname{dim}_{*}(m)
$$

which gives the non trivial inequality between these two numbers. Let us finaly remark that it is easy to prove that for almost every $x \in[0,1)$,

$$
\lim _{k \rightarrow+\infty} \frac{\log m\left(I_{T_{4 k}}(x)\right)}{-T_{4 k} \log 2}=s(p) .
$$

5. An extension of Theorems 1.1 and 1.2. The $\ell$-adic partition $\mathcal{F}_{n}$ of the cube $[0,1)^{D}$ is not the only situation where Theorems 1.1 and 1.2 make sense. Fix a sequence $\left(\ell_{n}\right)_{n \geq 1}$ of strictly positive real numbers and construct a family $\left(\mathcal{G}_{n}\right)_{n \geq 0}$ in such a way.

1. $\mathcal{G}_{0}=\left\{B_{0}\right\}$ where $B_{0}$ is a Borel set in $\mathbb{R}^{D}$ with $B(0,1 / c) \subset B_{0} \subset B(0, c)$ (we denote by $B(0, r)$ the ball with center 0 and radius $r$ ). 
2. For every $n \geq 0, \mathcal{G}_{n}$ is a finite family of disjoint Borel sets which are similar to $B_{0}$ in the ratio $\ell_{n}$.

3. For every $B \in \mathcal{G}_{n+1}$, there exists a unique $\tilde{B} \in \mathcal{G}_{n}$ such that $B \subset \tilde{B}$.

Let $m$ be a measure supported by a Borel set $E$ and suppose that for every $n \geq 0$, $E$ is a subset of $\bigcup_{B \in \mathcal{G}_{n}} B$. If we suppose that the sequence $\log \left(\ell_{n}\right) / n$ is bounded, then, coverings using elements of $\bigcup_{n} \mathcal{G}_{n}$ are sufficient to calculate the Hausdorff dimension of subsets of $E$. Conclusions of Theorem 1.1 and Theorem 1.2 are also true in this situation if we define $h_{*}(m)$ (resp. $\left.h^{*}(m)\right)$ as the lim inf (resp. lim sup) of the sequence

$$
h_{n}(m)=\frac{1}{\log \ell_{n}} \sum_{B \in \mathcal{G}_{n}} m(B) \log m(B) .
$$

In particular, our results can be applied for measures supported by Cantor sets constructed in the same way as those described in [1] or [2].

\section{REFERENCES}

[1] A. Batakis, Harmonic measure of some Cantor type sets, Ann. Acad. Sci. Fenn., 21 (1996), pp. 255-270.

[2] A. BATAKIS, A continuity property of the dimension of harmonic measure of Cantor sets under perturbations, Ann. Inst. H. Poincaré Probab. Statist., 36 (2000), pp. 87-107.

[3] A. BisBAS, A multifractal analysis of an interesting class of measures, Colloq. Math., 69 (1995), pp. 37-42.

[4] A. BISBAS AND C. KaRANIKAS, On the Hausdorff dimension of Rademacher Riesz products, Monatsh. Math., 110 (1990), pp. 15-21.

[5] G. Brown, G. Michon, and J. Peyrière, On the Multifractal Analysis of Measures, J. Stat. Phys., 66 (1992), pp. 775-790.

[6] L. CARLESON, On the support of harmonic measure for sets of Cantor type, Ann. Acad. Sci. Fenn., 10 (1985), pp. 113-123.

[7] K. FAlCONer, Fractal Geometry, Mathematical Foundations and Applications. John Wiley \& Sons Ltd., New-York, 1990.

[8] K. Falconer, Techniques in Fractal Geometry, John Wiley \& Sons Ltd., New-York, 1997.

[9] A.H. FAN, Sur la dimension des mesures, Studia Math., 111 (1994), pp. 1-17.

[10] A.H. FAN, K.S. LAU, AND H. RAO, Relationships between different dimensions of a measure, preprint.

[11] Y. Heurteaux, Estimations de la dimension inférieure et de la dimension supérieure des mesures, Ann. Inst. H. Poincaré Probab. Statist., 34 (1998), pp. 309-338.

[12] S.M. NGAI, A dimension result arising from the $L^{q}$ spectrum of a measure, Proc. Amer. Math. Soc., 125 (1997), pp. 2943-2951.

[13] M. TAMAShiro, Dimensions in a separable metric space, Kyushu J. Math., 49 (1995), pp. $143-162$.

[14] C. Tricot, Two definitions of fractional dimension, Math. Proc. Cambridge Philos. Soc., 91 (1982), pp. 57-74.

[15] L.S. Young, Dimension, entropy and Lyapounov exponents, Ergod. Th. \& Dynam. Sys., 2(1982), pp. 109-124.

[16] M. ZINSMEISTER, Formalisme thermodynamique et systèmes dynamiques holomorphes, volume 4 of Panoramas et synthèses, Société Mathématique de France, 1997. 